\title{
The evaluation of Candida albicans biofilms formation on silicone catheter, PVC and glass coated with titanium dioxide nanoparticles by XTT method and ATPase assay
}

\author{
Haghighi $\mathrm{F}^{1}$, Mohammadi ShR ${ }^{1}$, Mohammadi $\mathrm{P}^{2}$, Eskandari $\mathrm{M}^{3}$, Hosseinkhani $\mathrm{S}^{4}$ \\ Department of Medical Mycology, Tarbiat Modares University of Medical Sciences, Tehran, Iran. \\ sh.mohammadi@modares.ac.ir
}

\begin{abstract}
Lots of Candida albicans infections involve in biofilm formation on medical devices. This kind of biofilm can impede antifungal therapy and complicates the treatment of infectious diseases particularly in field of chronic diseases associated with implanted devices. This study has investigated the influence of treating silicone catheter, PVC and glass coated with Titanium dioxide (TiO2) nanoparticles on attachment of C. albicans. In this study $\mathrm{TiO} 2$ nanoparticles were synthesized from precursor $\mathrm{TiCl} 4$ and characterized by scanning electron microscopy (SEM) and X-ray diffraction (XRD) which showed TiO2 nanoparticles are 70-100 nm in size. In the simplest model of biofilms formation, C. albicans isolates (ATCC10231) and (ATCC 76615) were grown on the surface of small disks of catheter, PVC and glass in a flat-bottomed 12-well plates and evaluated biofilm formation using ATP bioluminescence and tetrazolium salt (XTT) reduction assays. In addition, morphology of $C$. albicans biofilms after $48 \mathrm{~h}$ incubation was observed by SEM. Results indicated that there is a statistical difference between mean of coated samples especially catheter and glass before and after TiO2 nanoparticles coating $(p<0.05)$. In SEM analysis, C. albicans biofilm was more aggregated on the surface of glass and catheter than PVC and control groups and after treatment by these nanoparticles, catheter and glass both showed most significant decrease of C. albicans attachment in comparison to the control groups (Fig. 4, Ref. 23). Full Text in PDF www.elis.sk. Key words: antifungal agent, biofilm, Candida albicans, coating, TiO2 nanoparticles.
\end{abstract}

Acknowledgements: This work was financially supported by Tarbiat Modares University of Tehran, Iran.

The incidence of mortality due to opportunistic microorganism has shown a marked increase in recent years. Candidiasis is one of the actually most ordinary fungal infections worlds widely (1). Currently C. albicans has more effective role among nosocomial pathogens due to proper potential for biofilm formation (2). Biofilms related medical device infections increase hospital patients and impose hospitalization costs. As the use and types of indwelling medical devices in modern healthcare are continuously expanding, especially with an aging population, the incidence of biofilms infections will be also rising. The main problem with microbial biofilms infections is their tendency to resist to the host immune system and all antimicrobial agents. In fact, counterparts of their free floating planktonic microbes within a biofilms are more resistant to antimicrobial agents $(3,4)$. Therefore, achiev-

${ }^{1}$ Department of Medical Mycology, Tarbiat Modares University of Medical Sciences, Tehran, Iran, ${ }^{2}$ Department of Biology, Faculty of Sciences, Alzahra University, Tehran, Iran, ${ }^{3}$ Nanomaterial Research Group, Academic Center for Education, Culture and Research of TMU, Tehran, Iran, and ${ }^{4}$ Department of Biophysics \& Biochemistry, Faculty of Biological Sciences, Tarbiat Modares University, Tehran, Iran

Address for correspondence: Sh.R. Mohammadi, PhD, Department of Medical Mycology, Tarbiat Modares University, Tehran, Iran.

Phone: +98.2182884540, Fax: +98.2182884555 ing a novel method to inhibit attachment of cells to the surface and eliminate of fungal mass over surfaces is valuable to control infections (5).

Nowadays great growing of nanotechnology in different branches of science such as engineering and medical is obviously noticeable. In recent years, nanomaterials have considerable acceptance to use as an antimicrobial effect due to different physical, chemical and electrical properties in ultra tiny form that unavailable in larger forms $(6,7)$.

In this study it was evaluated the influence of treatment of silicone catheter, PVC and glass coated using Titanium Dioxide (TiO2) nanoparticles on susceptible (ATCC 10231) and resistant (ATCC76615) standard strains of C. albicans strains.

These biofilms were analyzed through two viability cell techniques. First, XTT (Tetrazolium salt) assay as a colorimetric method and second one ATPase assay to assess biofilm biomass.

\section{Material and methods}

\section{1) Preparation of TiO2 nanoparticles}

TiO2 nanoparticles were synthesized through the hydrolysis of Titanium Tetrachloride (TiCl4) as precursor. In this step TiCl4 was slowly added into the $58 \mathrm{ml}$ distilled water under constant string for 5 hour. The solution was aged for $24 \mathrm{~h}$ at ambient temperature. Then gel was dried in oven for $12 \mathrm{~h}$ at $60{ }^{\circ} \mathrm{C}$ and finally calcinated at the $550{ }^{\circ} \mathrm{C}$. 


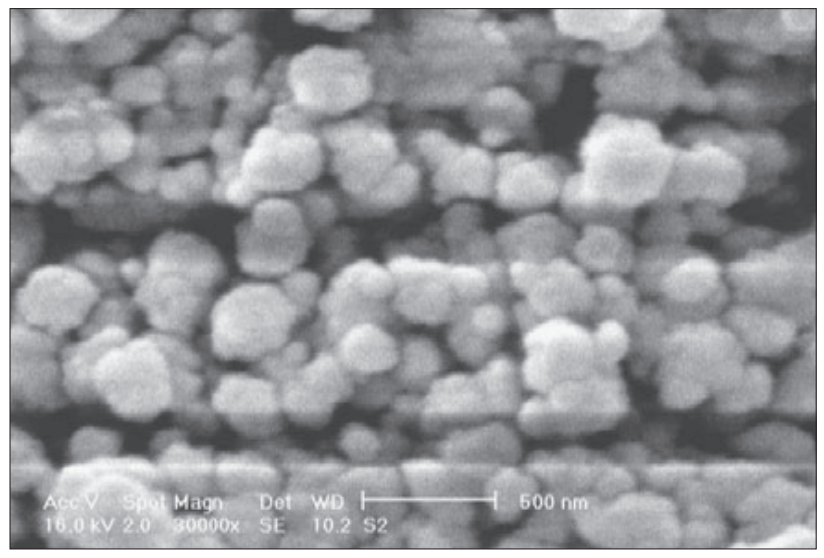

Fig. 1. SEM micrograph of TiO2 nanoparticles, Sizes of particles are 70-100 $\mathrm{nm}$ with rather uniform round shape.

\section{2) Characterising of TiO2 nanoparticles:}

a) Scanning Electron Microscopy (SEM) method,

b) To access information about shape and estimating size of nanoparticles, SEM (Philips) micrograph was taken Figure 1. After synthesize of $\mathrm{TiO} 2$ nanoparticles,

c) X-Ray Diffraction (XRD) method,

d) To identifying type of $\mathrm{TiO} 2$ nanoparticles, the synthesized TiO2 powder was characterised by XRD technique (XPERT: model 95) with k $\alpha$ radiation, wavelength $(\lambda=1.54178 \AA$ ) (Fig. 2), 3) Coating of catheter, PVC and glass by TiO2 nanoparticles.

Small pieces of silicone catheter, PVC and glass were cut in size of $1 \mathrm{~cm}^{2}$. Then surfaces of samples were washed with $\mathrm{NaOH}$ and they were finally coated with $\mathrm{TiO} 2$ nanoparticles by deep coating method. In this process, samples coated with continuous immersing and ejecting to the $\mathrm{TiO} 2$ nanoparticles solution.

4) Preparation of standard fungal cell suspension

Susceptible C. albicans (ATCC 10231) and resistant (ATCC 76615) standard strains of $C$. albicans was used to form biofilm and tested the antifungal activity of samples which was coated by

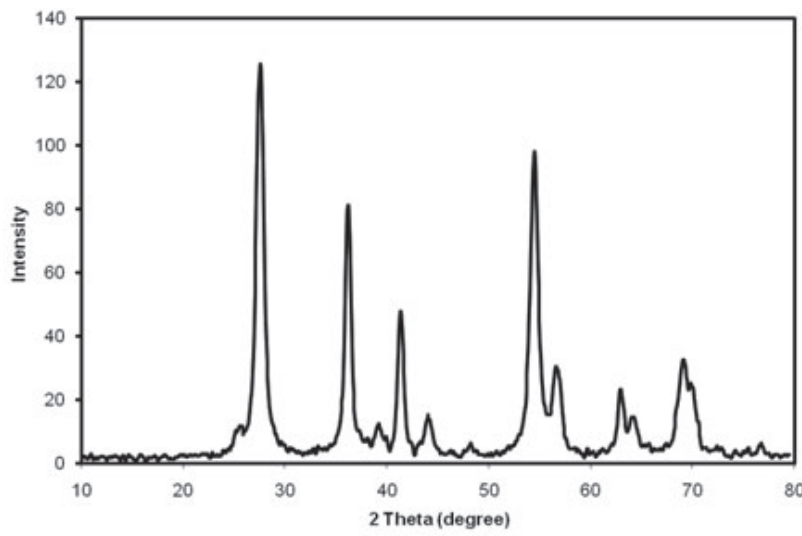

Fig. 2. XRD pattern of TiO2 nanoparticles with $\mathrm{Cu}$ Ka radiation at wavelength $\lambda=1.54178 \AA$.

$\mathrm{TiO} 2$ nanoparticles. At first, two strains were grown on sabouraud dextrose agar medium (SDA Merck, Germany) at $37^{\circ} \mathrm{C}$ for $18 \mathrm{~h}$. Then, freshly colonies inoculated into yeast nitrogen base medium (YNB medium; Himedia Co) containing $50 \mathrm{mM}$ glucose and incubated at $37^{\circ} \mathrm{C}$ for 24 hours. After that, little amounts of the yeasts were transferred into a test tube containing sterilized PBS with pH: 7.2 (difco). Next the mixture was centrifuged (10000 g, $10 \mathrm{~min}$ ) and turbidity of suspension of cells was compared to 0.5 McFarland standard tubes to estimate cell density. Finally yeast cells were counted and adjusted at $1 \times 10^{6}$ cells $/ \mathrm{ml}$ by using Neubauer slide.

\section{5) Biofilm formation}

According to Chandra et al (33) artificial candidal biofilm formation were carried out on 12-well tissue culture plates. Samples were placed in each well of 12 -well tissue culture plates. The yeast cells were incubated for $2 \mathrm{~h}$ at $37^{\circ} \mathrm{C}$ in $1 \mathrm{ml}$ of $1 \%$ bovine serum albumin (BSA). After this pretreatment, $80 \mu 1$ of C. albicans cell suspension was inoculated onto the surfaces. The yeast cells were allowed to adhere to the surface at $37{ }^{\circ} \mathrm{C}$ for $90 \mathrm{~min}$. After this
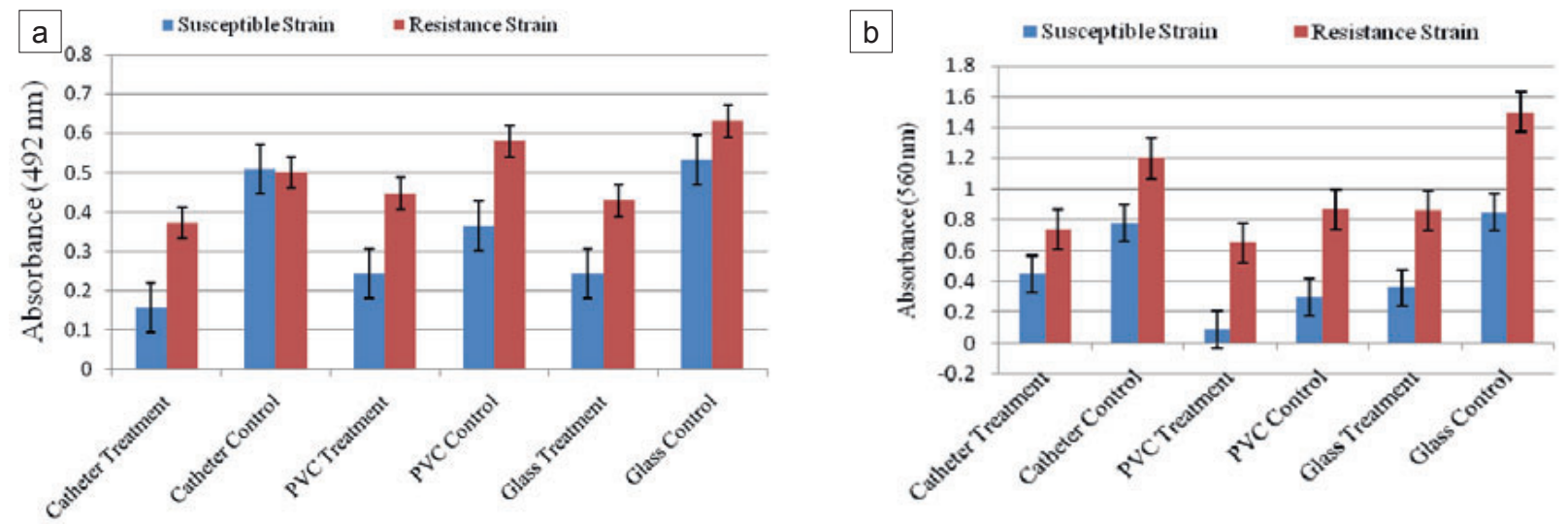

Fig. 3. Absorbance of $C$. albicans biofilms that formed at the surfaces of silicone catheter, PVC and glass disks and coated with TiO2 nanoparticles in comparison to control groups for both standard susceptible and resistance strains. The methods was used for biofilm quantification were XTT reduction assay (a), ATP bioluminescence assay (b). Treatment of Catheter, PVC and Glass also their control groups are represented in this figure. Data are means \pm standard deviations of three independent experiments performed in triplicate $(p<0.05)$. 

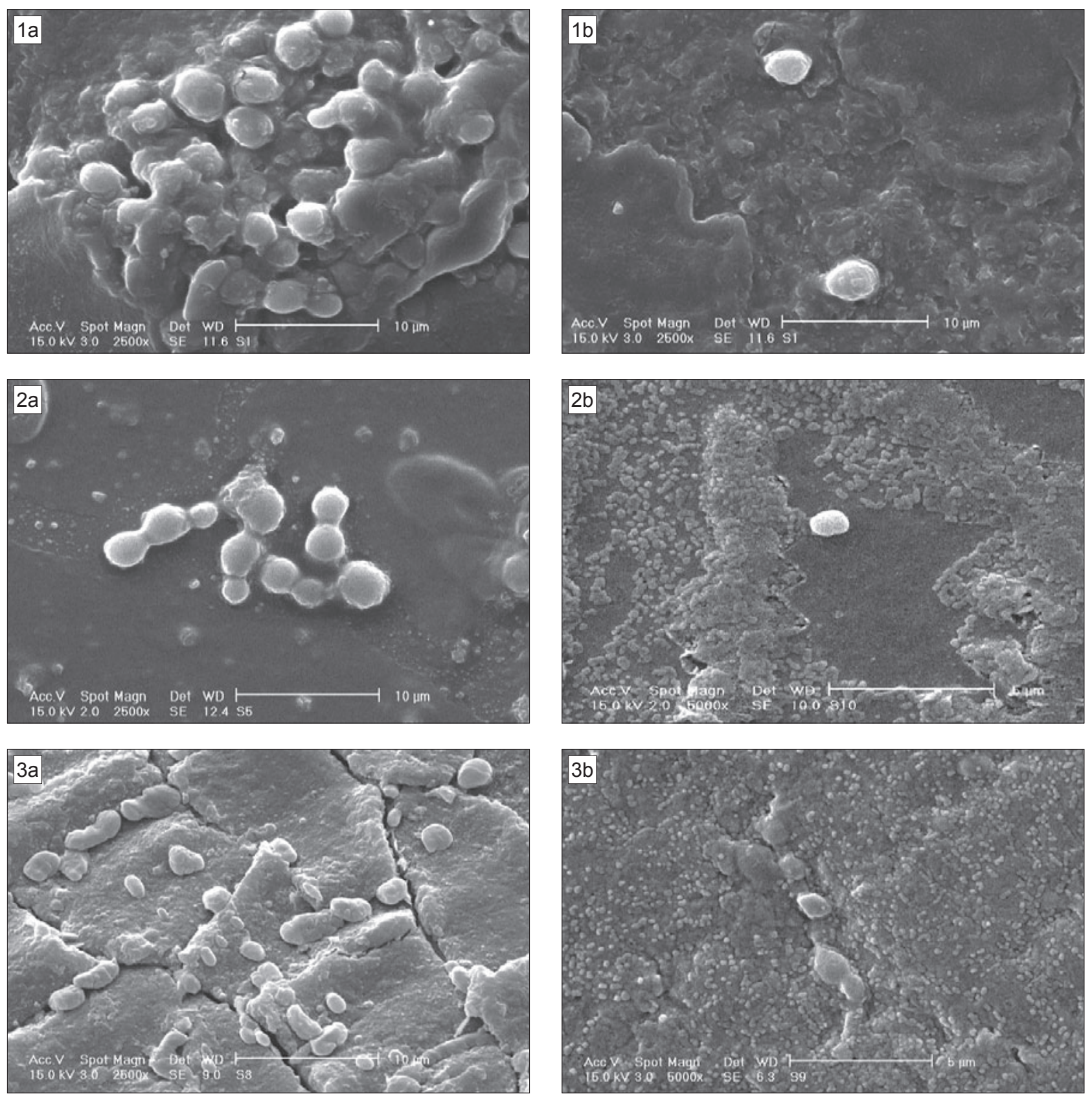

Fig. 4. SEM micrograph of biofilm formation on the surface of $1 \mathrm{~cm}^{2}$ pieces of glass (1), PVC (2), silicone catheter (3) before and after treatment with TiO2 nanoparticles, respectively (a and b). The best biofilm were formed at the glass and catheter surfaces.

time the slice of samples were gently washed with PBS and then added $4 \mathrm{ml}$ of YNB medium including $50 \mathrm{mM}$ glucose. The plates were incubated at $37^{\circ} \mathrm{C}$ for $48 \mathrm{~h}$, and the biofilms were formed on the surfaces. Afterward, the YNB medium was removed and washed with $4 \mathrm{ml}$ of PBS.

\section{6) XTT assay}

XTT [2, 3-bis (2-methoxy-4-nitro-5-sulfophenyl)-5- (phenyl amino) carbonyl)-2H-tetrazolium hydroxide] as colorimetric assay was carried out to evaluate antifungal effect of nanoparticles. This method is based on determining the viability of collected cells.
Antifungal assay was carried out by measuring the optical absorbance of incubated 96-wells plates containing $50 \mu 1$ of the collected cell suspension per well. Plates were incubated with $50 \mu \mathrm{l}$ of the YNB medium containing $50 \mathrm{mM}$ glucose and $100 \mu \mathrm{XTT}$ (Sigma) and $50 \mu \mathrm{l}$ coenzyme Q0 (Sigma) at $37^{\circ} \mathrm{C}$ for $3 \mathrm{~h}$. Optical absorbance (Fig. 3) was measured at the wavelength of $492 \mathrm{~nm}$ using an ELISA reader (Memmert, Germany).

7) ATPase assay

After biofilm formation and treatment of cells by nanoparticles, ATPase assay was used to determine the viability. For this reason, 
$707-711$

biofilm was removed by scrapping method, $10 \mu \mathrm{l}$ of susceptible and resistant of removed C. albicans, $10 \mu 1$ complex reagent containing luciferin and luciferase which was contained $\mathrm{Mg}^{+2}$, mixed in each well of Luminescence microplates. Then optical density was measured by luminometer (Berthold Co) at $560 \mathrm{~nm}$. ATP is a marker for cell viability. Because it is present in all metabolically active cells and its concentration declines very rapidly when the cells treated with the biocides. The ATPase assay system is based on production of light at $560 \mathrm{~nm}$ through the reaction of ATP with added luciferase and D-luciferin. All Data were analyzed by using statistical t-test method and SPSS software.

\section{Results}

Common morphology of the $\mathrm{TiO} 2$ nanoparticles is shown in Figure 1. It shows $\mathrm{TiO} 2$ nanoparticles which has regular shape.

The type of crystalline structure of the synthesized $\mathrm{TiO} 2$ nanoparticles has been considered by means of XRD with $\mathrm{Cu} \mathrm{Ka}$ radiation at wavelength $\lambda=1.54178 \AA$, and measurements were performed using a $\theta-2 \theta$ goniometry (8). Figure 2 shows XRD pattern $\mathrm{TiO}_{2}$ nanoparticles. All the diffraction peaks of samples show that synthesized $\mathrm{TiO} 2$ is the desired material. Crystal size of TiO2 nanoparticles are measured using Debye-Scherrer relation $[D=0.94 \lambda / \beta \cos (\theta)](8)$. It was observed that the diameter of the $\mathrm{TiO} 2$ nanoparticles is on average 70-100 nm (Fig. 2).

In this study, small pieces of glass and catheter that coated with $7.03 \mu \mathrm{g} / \mathrm{ml}$ of $\mathrm{TiO} 2$ nanoparticles showed reduction in cell density after coating of the samples with $\mathrm{TiO} 2$ nanoparticles. This reduction was more for susceptible standard strain of C. albicans when it was used XTT technique to assay.

ATPase assay results were according to XTT assay although; in comparison to control group differences was more than XTT method. All experiments were performed in triplicate and three independent experiments $(\mathrm{p}<0.05)$.

Results of treated samples using scanning electron microscope showed that attached $C$. albicans to treated glass and catheter surfaces significantly decreased in comparison to control groups.

\section{Discussion}

Human tissues frequently have large and compound microbial communities that are growing as biofilm and according to the condition cause a variety of infections. As a result of greater use of implanted medical devices than before, microbes in biofilm display increased resistance towards biocides; antibiotic chemotherapy as well decreased susceptibility to the host defense mechanisms $(9,10)$. Recent evidences have revealed that in more than $65 \%$ microbial infection, biofilms have critical role in infections and diseases associated biofilm and are becoming increasingly more difficult to treat (11). Candida is an important human fungal flora causing nosocomial infection. Candida species have capability to adhere to many surfaces and form biofilms. The surfaces of medical devices supply a substrate for colonization of microbes. The evidence linking Candida biofilms of medical devices associated with infections is growing as more consistent way for evaluating Candida biofilms (12).
Many studies have been investigated about eradicating the biofilm populations using variety of conventional drugs and antimicrobial agents to eliminate fungi in order to control unfavorable biofilm surfaces and in aqua environments by using antifungal biocides or UV radiation (13). The function of electronic microscopy and molecular methods has developed our understanding about biofilm structure and attachment of this structure to achieve important information about the prevention and treatment of biofilm formation on a variety of surfaces, important to the medical practices till industries which are useful to develop preventive strategies and methods for biofilm control (14). According to expensive and consuming treatment of infection disease and difficulty in treatment as well genetic variation and increasing drug resistance, introducing agents that possess antifungal properties and inhibitory biofilm formation could be effective role in three stages of controlling, prevention and treatment of disease associated biofilms (15).

Because of the great ability of nanoparticles in coating surfaces and regard to antimicrobial activity of these nanoparticles, we used $\mathrm{TiO} 2$ nanoparticles to coating silicone catheter, PVC and glass for analyzing C. albicans biofilm formation. According to Akiba (16) and Chen (17) studies, nanoparticles such as $\mathrm{TiO} 2$ have antimicrobial efficacy which could be considered as a self-cleaning agent.

According to the antifungal properties of $\mathrm{TiO} 2$ nanoparticles in amount of $4 \mu \mathrm{g} / \mathrm{ml}$ for susceptible and $4.55 \mu \mathrm{g} / \mathrm{ml}$ for resistance strain (Haghighi and colleagues in 2010) (18) in this study it was tried to use three different surfaces, silicone catheter, PVC and glass as a substratum for biofilm formation.

The results of XTT method and ATPase assay on biofilm formation showed glass and catheter coated with $\mathrm{TiO} 2$ nanoparticles had better ability to inhibit attachment, and formed biofilm was more for PVC surface (Fig. 4).

Studies by many laboratories have showed that microbial attachment to the treated PVC were stronger than latex and silicone surfaces because of some surface properties such as texture, charge and hydrophobicity. The catheters with rough, irregularities surface show considerably more adherent compared to catheters with smoother surfaces $(19,20)$.

It was proved by scientists that $\mathrm{TiO} 2$ nanoparticles, producing intracellular reactive oxygen species (ROS), induced destructive effects inside the microbial cells and oxidation of intra cellular Coenzyme $\mathrm{A}(\mathrm{Co} \mathrm{A})$ and peroxidation of plenty lipids which is resulted decreased respiratory activity and consequently cell death $(21,22)$.

We evaluated the amount of viability of sessile cells of biofilms by using XTT assay. Furthermore, results of XTT assay have accordance with ATPase analyses. The major advantages of ATPase assay compared to conventional methods for viability detection are high sensitivity, excellent linearity, simplicity and fast to do and with lack of cell harvesting or separation steps without any need to incubate the cells (23).

This study showed that $\mathrm{TiO} 2$ nanoparticles able to inhibit $C$. albicans biofilms formation. Consequently in field of controlling and elimination of C. albicans biofilms, $\mathrm{TiO} 2$ nanoparticles can be considered as an alternative antifungal agent although further investigation related to optimizing $\mathrm{TiO} 2$ nanoparticles synthesize and procedure of coating surfaces must be done. 


\section{Conclusion}

In this study, it was shown that $\mathrm{TiO} 2$ nanoparticles inhibit the growth of candida biofilms. And this inhibitory effect was more for the glass and then catheter. According to this results ATPase assay can achieve fast and more reliable results thus consuming less time.

\section{References}

1. Sardi JC, Duque C, Mariano FS, Peixoto IT, Hofling JF, Gonçalves RB. Candida spp. in periodontal disease: a brief review. J Oral Sci 2010; 52 (2): $177-185$.

2. Nett J, Andes D. Candida albicans biofilm development, modeling a host-pathogen interaction. Curr Opin Microbiol 2006; 9 (4): 340-345.

3. Nett JE, Andes D. Review of Techniques for diagnosis of catheter-related candida biofilm infections. Current Fungal Infec Rep 2008; 2: 237-243.

4. Jabra-Rizk MA, Falkler WA, Meiller TF. Fungal Biofilms and Drug Resistance. Emerg Infect Dis 2004; 10 (1): 14-19.

5. Butterfield PW, Bargmeyer AM, Camper AK, Biederman JA. Modified enzyme activity assay to determine biofilm biomass. J Microbiol Methods 2002; 50 (1): 23-31.

6. Jiang W, Mashayekhi H, Xing B. Bacterial toxicity comparison between nano- and micro-scaled oxide particles. Environ Pollut 2009; 157 (5): 1619-1625.

7. Zhang L, Ding Y, Povey M, York D. ZnO nanofluids - A potential antibacterial agent. Progress in Natural Science 2008; 18 (8): 939-944.

8. Arabi AM, Ebadzadeh T, Yousefi AA, Pishvaei M, Marzban RadE, Najafi F. ZnO nanofluids: Green synthesis, characterization, and antibacterial activity. The function of nano-polystyrene template and comb polycarboxylic acid surfactant in synthesis of $\mathrm{ZnS}$ nanoparticles via hydrothermal method. Ir Polymer J 2011; 20 (7): 559-569.

9. Nett JE, Sanchez H, Cain MT, Ross KM, Andes DR. Interface of Candida albicans Biofilm Matrix-Associated Drug Resistance and Cell Wall Integrity Regulation. Eukaryo Cell 2011; 10 (1): 5126-5111.

10. Prakash B, Veeregowda BM, Krishnappa G. Biofilms: A survival strategy of bacteria. Curr Sci 2003; 85 (9): 1299-1307.

11. Ferreira JA, Carr JH, Starling CE, de Resende MA, Donlan RM. Biofilm formation and effect of caspofungin on biofilm structure of Candida species bloodstream isolates. Antimicrob Agents Chemother 2009; 53 (10): 4377-4384.
12. Uppuluri P, Chaturvedi AK, Srinivasan A, Banerjee M, Ramasubramaniam AK, Kohler JR, Kadosh D, Lopez-Ribot JL. Dispersion as an important step in the Candida albicans biofilm developmental cycle. PLoS Pathog 2010; 6 (3): 1000-1828.

13. Theraud M, Bdouin Y, Guiguen C, Gangneux JP. Efficacy of antiseptics and disinfectants on clinical and environmental yeast isolates in planktonic and biofilm conditions. J Med Microbiol 2004; 53 (10): 1013-1018.

14. Sasidharan S, Yoga Latha LY, Angeline T. Imaging In vitro Antibiofilm Activity to Visualize the Ultrastructural Changes. Microscopy: Scie Tech App Edu 2010; 15 (2): 622-626.

15. Nett JE, Sanchez H, Cain MT, Andes DR. Genetic basis of Candida biofilm resistance due to drug-sequestering matrix glucan. J Infect Dis 2010; 202 (1): 171-175.

16. Akiba N, Hayakava I, Keh ES, Watanabe A. Antifungal effect of a tissue conditionar coatingagent with $\mathrm{TiO} 2$ photocatalyst. J Med Dent Sci 2005; 52: 223-227.

17. Chen Y, Yan L, Wang R, Fan H, Zhang Q. Antimicrobial Polyurethane Synthetic Leather Coating with In-situ Generated Nano-TiO2. Fiber polymer 2010; 11 (5): 689-694.

18. Haghighi F, Roudbar Mohammadi Sh, Mohammadi P, Eskandari M. The evaluation of antifungal activity of titanium dioxide nanoparticles and ethylene diamine tetra acetic acid on growth inhibition standard strain of Candida albicans. Quarter J Yasuj Uni Ir 2010; 15 (2): 134-141.

19. Darouiche RO, Device-associated infections: a macroproblem that starts with microadherence. Clin Infect Dis 2001; 33 (9): 1567-1572.

20. Pascual A, Pathogenesis of catheter-related infections: lessons for new designs. Clin Microbiol Infect 2002; 8 (5): 256-264.

21. Battin TJ, Kammer FVD, Weilhartner A, Ottofuelling S, Hofmann T. Nanostructured $\mathrm{TiO}_{2}$ : Transport Behavior and Effects on Aquatic Microbial Communities under Environmental Conditions. Environ Sci Technol 2009; 43 (21): 8098-8104.

22. Foster HA, Ditta IB, Varghese SA. Photocatalytic disinfection using titanium dioxide: spectrum and mechanism of antimicrobial activity. Applied Microbiol Biotech 2011: 90 (6); 1847-1868.

23. Pihlasalo S. Quantification of Proteins and Cells: Luminometric Nonspecific, A thesis presented for degree of PHD. Department of Cell Biology and Anatomy, Annales Universitatis Turkuensis of Turku Finland. 2011.

Received November 12, 2011. Accepted January 17, 2012. 